\title{
ORIGINAL ARTICLE \\ Early spermatogenesis changes in traumatic complete spinal cord-injured adult patients
}

\author{
A Sánchez-Ramos ${ }^{1,2}$, E Vargas-Baquero ${ }^{1,2}$, FJ Martin-de Francisco ${ }^{3}$, JA Godino-Durán ${ }^{4}$, I Rodriguez-Carrión ${ }^{4}$, \\ M Ortega-Ortega ${ }^{1}$, L Mordillo-Mateos ${ }^{5}$, F Coperchini ${ }^{6}$, M Rotondi $^{6}$, A Oliviero ${ }^{5}$ and M Mas ${ }^{2,7}$
}

Study design: Prospective longitudinal study.

Objectives: To assess the impact of spinal cord injury (SCI) on the spermatogenesis of patients studied in the early subacute stage and ensuing months.

Setting: National hospital for $\mathrm{SCl}$ patients.

Methods: A prospective follow-up study was conducted on 28 male patients with complete $\mathrm{SCl}$ who were evaluated in the early subacute phase ( 1 month), and 3 and 6 months after the injury. At each time point, fine needle aspiration biopsy samples were taken from the testes for cytological assessment, and serum levels of relevant hormones were measured. At the end of the study period, ejaculation was attempted for standard semen analyses.

Results: Cytological patterns indicative of defective spermatogenesis were found in $61 \%, 52 \%$ and $20 \%$ of the patients at 1, 3 and 6 months after $\mathrm{SCl}$, respectively, suggesting an improvement over time. Serum hormone analyses showed a steady elevation of estradiol levels above the reference range, and increasing levels of testosterone, inhibin B and prolactin throughout the study period. Prolactin levels were above the reference range at all time points. Only 2 out of the 10 patients who were able to ejaculate at 6 months post injury showed normal sperm parameters.

Conclusion: A majority of the patients showed impaired spermatogenesis soon after the injury, which in most cases recovered over time. That was accompanied by parallel increases in serum levels of inhibin B, testosterone and prolactin, possibly driving or reflecting the spermatogenesis recovery. Further studies are needed to elucidate the biological mechanisms underlying these changes.

Spinal Cord (2017) 55, 570-574; doi:10.1038/sc.2016.184; published online 24 January 2017

\section{INTRODUCTION}

Men with spinal cord injury (SCI) have impaired sexual health and fertility, namely, erectile dysfunction, ejaculatory dysfunction and poor semen quality. ${ }^{1-3}$ In anejaculatory cases of SCI, sperm may be retrieved using penile vibratory stimulation, electroejaculation (EEJ) or surgical sperm retrieval. ${ }^{4-6}$ The availability of assisted reproductive techniques allows most men with SCI to conceive potentially.

Traumatic SCI patients, typically young men, represent the largest physical medicine and rehabilitation population with fertility-related disorders with clear consequences on both quality of life and social costs. Although $3-20 \%$ of these men can ejaculate with self-stimulation, this ability tends to be unpredictable and it is rarely effective for achieving conception. ${ }^{1-3}$ However, even when semen is obtainable (by self-stimulation or rectal probe EEJ), azoospermia is not uncommon and asthenozoospermia is found in a considerable percentage of patients. ${ }^{6}$ Brackett $e t$ al. demonstrated that the seminal plasma of SCI men could be detrimental to spermatozoa vitality. ${ }^{7}$ Nevertheless, the causes of poor semen quality of SCI patients are still unclear as several factors, some of them potentially reversible, seem to be involved. ${ }^{2,8-10}$
Besides genetic and ethnic differences, ${ }^{8,11}$ hormonal changes were reported to exert potentially relevant effects on spermatogenesis. ${ }^{12,13}$

To our knowledge, the spermatogenic function has not been previously evaluated in the days/months immediately after the SCI in humans. There are reports, however, of spermatogenesis abnormalities in the subacute phase of SCI in animal models. ${ }^{14,15}$ As the accepted duration of human spermatogenesis, including spermatogonia proliferation, is 74 days ( $95 \%$ confidence interval (CI): 69-80 days), ${ }^{16}$ we considered that the first 6 months would be an appropriate time frame for evaluating the early repercussion of a SCI on spermatogenesis.

The spermatogenic function can be evaluated cytologically by testis biopsies obtainable with the minimally invasive technique of fine needle aspiration (FNA). This procedure is widely used to examine pathological changes in various human organs. Currently, FNA cytology (FNAC) represents an alternative to open testicular biopsy for characterizing human male infertility owing to defective spermatogenesis. FNAC has gained popularity as a diagnostic tool for the assessment of male infertility for several reasons including the relatively uniform cellularity of the testis and the possibility of

${ }^{1}$ Department of Rehabilitation, Sexual Medicine Unit, Hospital Nacional de Parapléjicos, Toledo, Spain; ${ }^{2}$ Center for Sexological Studies (CESEX), Universidad de La Laguna, Tenerife, Spain; ${ }^{3}$ Department of Pathology, Hospital Virgen de la Salud, Toledo, Spain; ${ }^{4}$ Clinical Neurophysiology Unit, Hospital Nacional de Parapléjicos, Toledo, Spain; ${ }^{5}$ FENNSI Group, Hospital Nacional de Parapléjicos, Toledo, Spain; ${ }^{6}$ Unit of Internal Medicine and Endocrinology, Fondazione Salvatore Maugeri IRCCS, Laboratory for Endocrine Disruptors and University of Pavia, Pavia, Italy and ${ }^{7}$ Department of Basic Medical Science, Physiology Unit, Universidad de La Laguna, Tenerife, Spain

Correspondence: Dr A Sánchez-Ramos, Department of Rehabilitation, Sexual Medicine Unit, Hospital Nacional de Parapléiicos, Finca La Peraleda s/n, 45071 Toledo, Spain. E-mail: asanchezramos@telefonica.net

Received 16 July 2016; revised 17 November 2016; accepted 24 November 2016; published online 24 January 2017 
repeating the procedure with a high safety profile. Most important, the results of testis FNAC analyses show good concordance with classic histological biopsy data. ${ }^{17-20}$

We choose to evaluate FNAC to assess spermatogenesis rather than sperm analysis (with sperm obtained by ejaculation induction) for two main reasons. First, in the subacute phase, it is unlikely to recruit SCI men for a semen collection via rectal probe stimulation. Moreover, in the early subacute phase, the sperm collection by stimulation owing to the spinal shock (specially using vibrators) is difficult. Second, there is evidence of a significant contribution from extratesticular factors to the poor sperm quality found in SCI patients. ${ }^{2,7,21-22}$ These extratesticular factors are ruled out using FNAC. Thus, we decided to use that approach to explore early changes in spermatogenesis patterns and their possible relationship with blood levels of reproductive hormones following SCI in men.

\section{PATIENTS AND METHODS}

A group of 28 male patients (mean age $34.9 \pm 8.9$ (s.d.), range 19-41) bearing a recent SCI were assessed prospectively. The patients were evaluated in the early subacute phase ( 1 month post SCI, 'baseline'), and then 3 and 6 months after the injury.

\section{Patients}

The patients included for the study had subacute traumatic SCI with a lesion level between $\mathrm{C} 4$ and T12. The mean time from the injury to the first evaluation was 28.1 days \pm 6.0 (s.d.). To have a relatively homogenous group of subjects, the study was restricted to SCI patients with complete lesions. They all were selected on the basis of having an AIS A grade of the ASIA Impairment Scale, ${ }^{23}$ and complete absence of both somatosensory potentials evoked by pudendal nerve stimulation and bulbocavernous muscle motor potentials following transcranial magnetic stimulation. ${ }^{24}$ The patient's lesion levels were cervical (C4-C8, $n=6$ ), thoracic (T1-T9, $n=13$ ) and thoraco-lumbar (T10-L1, $n=9$ ). The study was conducted in accordance with the Declaration of Helsinki and was approved by the institutional ethics committee. All subjects provided written informed consent. Patients were not informed about results before the end of the study (for example, at month 6).

\section{Testis biopsy}

During the FNA procedure, the patients received no anesthesia owing to the completeness of their spinal cord lesion. FNAC samples were drawn using a $20 \mathrm{ml}$ syringe with an insulin needle mounted in a 'CAMECO Universal' syringe gun holder. The procedure was well tolerated, and there were no side effects. The FNA cytological smears were stained with H\&E and examined by an experienced cytopathologist who was unaware of the patient's time-group allocation. The aspirates were considered adequate when at least 300 cells could be counted in at least one well-spread smear. Different cells in FNAC smears were identified by their nuclear and cytoplasmic characteristics, following previous descriptions. ${ }^{17-20}$ The percentage populations of Sertoli cells and various spermatogenetic cells, including spermatogonia, spermatocytes, spermatids and spermatozoa, were calculated. Several combinations of those values provide a number of indices deemed useful to differentiate between various types of spermatogenesis defects. ${ }^{18}$ The spermatic index is calculated as the ratio of spermatozoa to total spermatogenetic cells (including spermatozoa); it reflects the efficacy of spermiogenesis. The Sertoli cell index (SerCI: ratio of Sertoli cells to all spermatogenetic cells, including spermatozoa) is inversely related to spermatogenesis, whereas the sperm-SerCI (SSerCI: ratio of spermatozoa to Sertoli cells) is directly related to it. Thus, hypospermatogenesis is characterized by low SSerCI but normal spermatic index, in addition to low spermatozoa percentage. The maturation arrest pattern is fairly similar except for a much reduced spermatic index. ${ }^{18}$ On the basis of the standard cytopathological interpretation of the H\&E smears, the biopsies would be classified accordingly into six diagnostic groups: (1) Sertoli cell-only (not found in our patients); (2) maturation arrest, (3) severe hypospermatogenesis, (4) moderate hypospermatogenesis, (5) mild hypospermatogenesis, (6) normal spermatogenesis. Hypospermatogenesis indicates that all stages of spermatogenesis, including mature sperm, are present, but the relative number of cells is decreased.

\section{Semen collection and analysis}

Semen collection was attempted in all of the patients at 6 months post injury. First, we tried vibratory stimulation (VS) with the FertiCare vibrator (Multicept A/S, Frederiksberg, Denmark). When proved ineffective, EEJ was induced by stimulation with a rectal probe (STIMED Handisoft, Chalon sur Saone, France) to deliver an increasing series of duplicate voltage pulses ( $4 \mathrm{~s}$ each) with $2 \mathrm{~V}$ increments until ejaculation occurred. ${ }^{2,21,22,25-28}$ Antegrade semen specimens were allowed to liquefy for $30-40 \mathrm{~min}$ at room temperature. Semen specimens were analyzed for volume, sperm concentration and sperm motility. Sperm motility was graded according to the WHO criteria as a-rapid linear motility, b-sluggish motility, c-nonprogressive motility and d-immotility. Sperm morphology data were not analyzed in this study. ${ }^{29}$

\section{Hormone assays}

At each time point, a blood sample was drawn to measure the serum levels of follicle-stimulating hormone, luteinizing hormone, testosterone, prolactin, estradiol and inhibin B (ELISA assays).

\section{Data analysis}

The changes in the proportion of patients showing the different spermatogenetic patterns at the three time points studied were assessed by using the Cochran-Armitage method $\left(\chi^{2}\right.$-test for trend). All the continuous variables studied were first inspected for adjustment to the normal distribution using the D'Agostino-Pearson test. As most of the cytological indices did not pass it, the nonparametric Friedman test for repeated measures was used to check for changes between the three time intervals studied. When significant, paired post hoc comparisons were made with the Dunn test. The serum hormone concentrations at the different time points passed the normality test. Hence, repeated measures analysis of variance followed by post hoc comparisons with their initial values (baseline, 1 month post SCI) by the Dunnett method as well as linear trend analyses were used to assess changes over time.

The significance level was set at $P<0.05$ in all analyses. The GraphPad Prism v.6 software (GraphPad Software, La Jolla, CA, USA) was used for statistical analyses and graphics.

\section{RESULTS}

\section{Cytological data}

No side effects were observed in any of the FNA procedures. At baseline ( 1 month post SCI), adequate FNA specimens (that is, valid for cytopathological evaluation) were obtained from all the patients studied. At baseline, spermatogenesis was normal in 11/28 men (39\%) and hypospermatogenesis was diagnosed in $16(57 \%)$; it was mild in $6 / 28(21 \%)$, moderate in $3(11 \%)$ and severe in 7 (25\%). Besides this, one patient (4\%) had maturation arrest at this time. At 3 months post injury, adequate FNA samples were obtained from 25 patients (three dropouts $)-12(48 \%)$ showing a normal spermatogenesis pattern, whereas 13 (52\%) had hypospermatogenesis. By 6 months post injury, valid smears were obtained from 21 patients-17 (80\%) had normal spermatogenesis with only 4 patients (20\%) still showing hypospermatogenesis (Figure 1). There was a significant positive trend over time in the proportion of subjects with normal spermatogenesis $\left(\chi^{2}\right.$-test for trend: $\left.P<0.005\right)$.

The changes in the cytological indices are summarized in Figure 2. Friedman tests for repeated measures detected significant overall differences for several of the indices studied. Thus, there were significant increases in spermatozoa percentage and SSCI, and a significant decrease in Sertoli cell counts. Post hoc analyses with the Dunn's paired comparisons test confirmed significant differences 


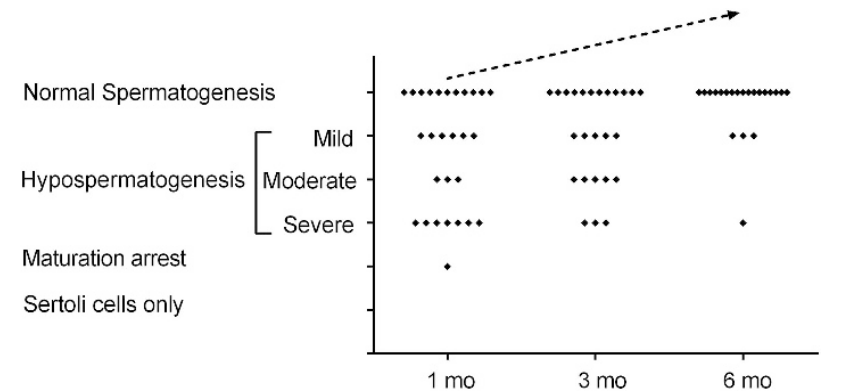

Figure 1 Cytological patterns observed in FNA smears from $\mathrm{SCl}$ patients at different time points post injury: 1 month (baseline), and 3 and 6 months. The dashed arrow indicates a significant positive trend in the proportion of subjects with normal spermatogenesis $\left(\chi^{2}\right.$-test for trend: $\left.P<0.005\right)$.

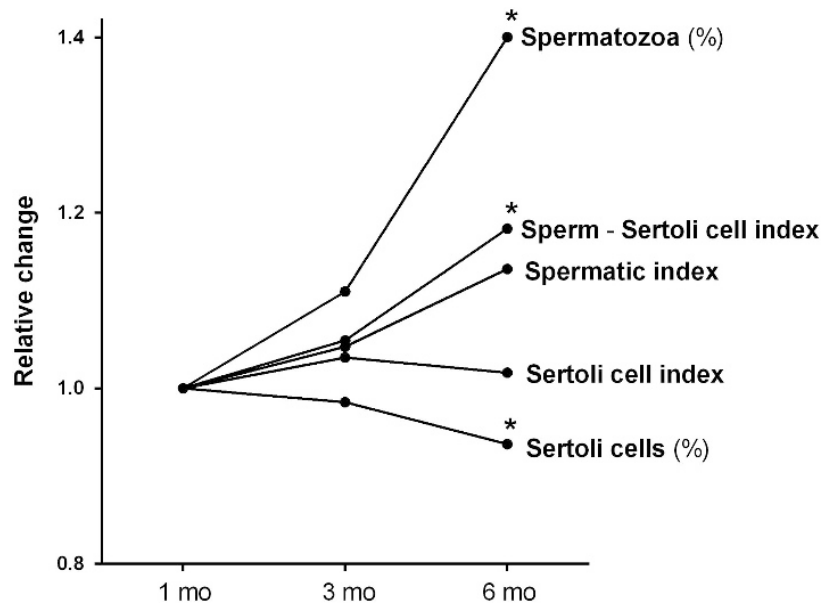

Figure 2 Trends in cytological indices in FNA biopsy smears taken at different intervals following $\mathrm{SCl}$. The changes are shown as the mean ratio to the initial value (baseline $\sim 1$ month post $\mathrm{SCl}$ ). Asterisks denote significant differences $(P=0.01-<0.05)$ with baseline. See text for indices explanation.

between their values at 6 months post SCI and baseline (1 month values), with $P$-values ranging from $0.01-<0.05$.

The cytological patterns observed in FNA smears from patients with thoraco-lumbar SCI level showed a tendency to be worse than the thoracic SCI group (thoracic SCI group $6 \pm 1.3, n=13$; thoracolumbar SCI group $5 \pm 1.5 n=9$; Mann-Whitney test $P>0.05)$. Because of the small sample size $(n=6)$, the patients with a cervical lesion level were not included in this comparison.

\section{Hormone levels}

At baseline, follicle-stimulating hormone, luteinizing hormone, testosterone and inhibin $\mathrm{B}$ were within the lab normal reference range, whereas estradiol and prolactin were above it at all time points (Figure 3). Repeated measures analysis of variance detected significant overall differences for prolactin, testosterone and inhibin B values. Their levels increased steadily over time, as evidenced by post hoc comparisons with the baseline values (1 month post SCI) with Dunnet's test and by significant linear trends analyses (Figure 3).

\section{Semen analysis}

Six months after the injury, ejaculation induction was attempted in 22 patients. In 18 subjects, ejaculation was attempted using VS only, and in only 4 using EEJ after VS failure. Only 10 (45\%) patients were able to ejaculate ( 6 using VS and 4 needed EEJ). Semen was obtained in small volume $(1.5 \pm 0.85 \mathrm{ml}$, mean \pm s.d.; range $0.1-2.8 \mathrm{ml})$ as compared with our own normal reference range. Also sperm concentration was lower than normal $\left(74.4 \pm 63.3 \times 10^{6}\right.$ per $\mathrm{ml}$; range 6-192 $\times 10^{6}$ per $\left.\mathrm{ml}\right)$. Sperm motility types were as follows: $a=6.2 \pm 5.3 \% ; b=7.3 \pm 6.2 \% ; c=7.9 \pm 6.2 \%$ and $d=77.4 \pm 14.6 \%$. Total motility $(a+b+c)$ was $21.4 \pm 12 \%$ and progressive motility $(a+b)$ was $13.6 \pm 8.9 \%$. When individually analyzed, only two patients had normal values for sperm quantity and quality.

\section{DISCUSSION}

This is the first prospective study describing early changes in spermatogenesis after complete SCI in humans and their evolution during the ensuing months. We found impaired spermatogenesis in a majority of the patients soon after the injury (within 30 days). Thereafter, spermatogenesis recovered spontaneously being apparently normal in most of the patients by 6 months following the SCI. The spermatogenesis improvement was reflected in the progressive increase in the percentage of spermatozoa, spermatic index and SSerCI, and a corresponding decrease in the \% of Sertoli cells in the FNAC samples over time.

Only 10 patients were able to ejaculate at 6 months after the injury (using vibrostimulation or electrostimulation). In other words, only $45 \%$ of the patients were able to ejaculate at a time post SCI, when $80 \%$ of the patients had normal testis biopsies. This is not surprising because ejaculation and sperm production are notoriously unrelated phenomena. Moreover, only two of those patients (20\%) had a quality and quantity of sperm that could be regarded as normal. This finding would be in line with the evidence of a significant contribution from extratesticular factors to the poor sperm quality found in SCI patients. ${ }^{2,7,21-22}$

Hormone analyses showed increased levels of estradiol and prolactin at baseline. High estradiol levels remained stable, whereas prolactin increased further along the study period. Although testosterone and inhibin levels were both within the lab reference range at all time points, they increased progressively from baseline to 6 months post SCI.

Thus, the SCI seems to damage the spermatogenetic function immediately after the injury (within 30 days), then recovering during the few ensuing months. Likewise, the parallel increases in testosterone levels indicate that the testis endocrine function is also working better over time. Furthermore, inhibin B changes over time could reflect the improved spermatogenesis. Whether both phenomena are causally related cannot be answered with the present data, however. In any case, these findings are suggestive of spinal shock phenomena at the testis level.

High prolactin levels have been found, inconsistently, in some of the patients bearing chronic SC lesions. ${ }^{30,31}$ It was not surprising that our patients had high prolactin levels by 1 month after the injury, probably as a part of the stress response to it. However, the progressive increase of prolactin over time was unexpected, as the patients were not taking drugs known to increase prolactin secretion. Therefore, an alternative explanation is needed for the progressive increase in prolactin levels.

The parallel time course of increasing prolactin levels and spermatogenesis recovery might reflect a causal relationship. Indeed prolactin receptors are expressed in the human testis by differentiating germ 
FSH

$(\mathrm{mU} / \mathrm{ml})$

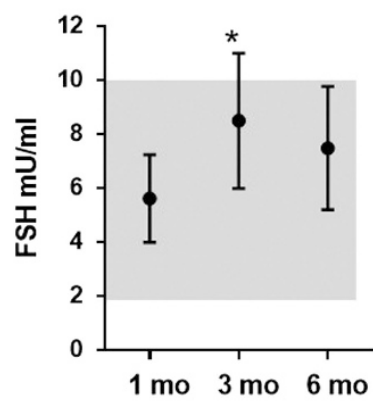

Testosterone (ng/ml)

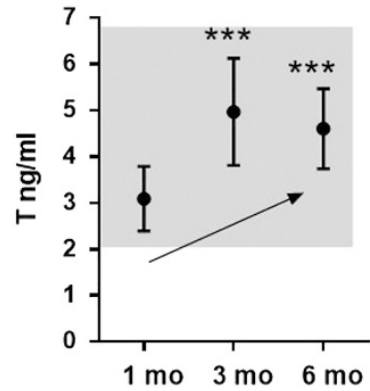

LH

$(\mathrm{mU} / \mathrm{ml})$

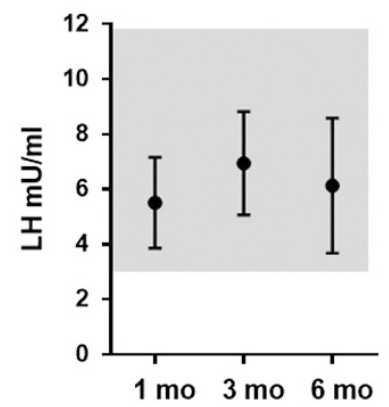

Estradiol

$(\mathrm{pg} / \mathrm{ml})$

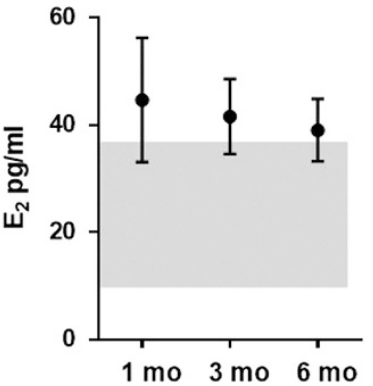

Prolactin

(ng/ml)
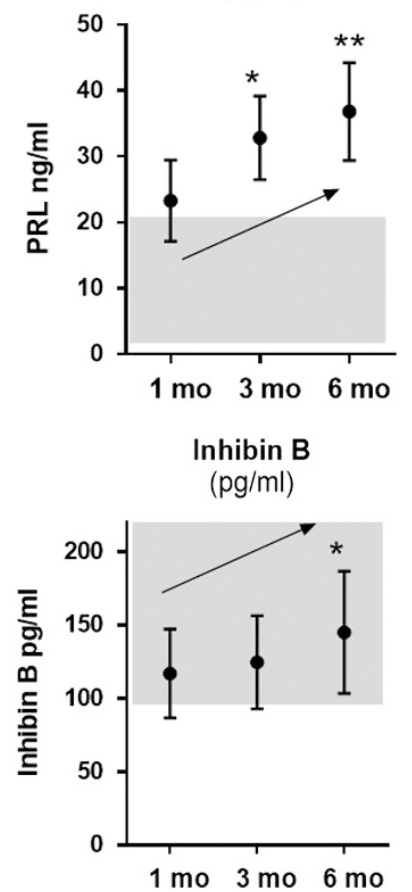

Figure 3 Changes in serum hormone levels following SCl. The plotted values are the means $\pm 95 \% \mathrm{Cl}$. The shaded areas show the reference range for ablebodied, presumably fertile men. Significance vs baseline levels ( 1 month post $\mathrm{SCl}$ ): ${ }^{*} P<0.01 ;{ }^{*} P<0.001 ;{ }^{* *} P<0.0001$. The arrows indicate significant linear trend $(P<0.001)$. See Figure 1 legend for further details. FSH, follicle-stimulating hormone; LH, luteinizing hormone.

cells (from pachytene spermatocytes to elongating spermatids) as well as by Leydig cells. ${ }^{32}$ Future longitudinal studies carried out on a large series of SCI patients will be required to confirm this result, as well as to clarify whether higher prolactin levels have a role in mantaining normal spermatogenetic function. In addition, the transient increase in follicle-stimulating hormone at 3 months post SCI and the stable higher levels of estradiol also appear potentially relevant for the observed changes in spermatogenesis.

\section{DATA ARCHIVING}

There were no data to deposit.

\section{CONFLICT OF INTEREST}

The authors declare no conflict of interest.

\section{ACKNOWLEDGEMENTS}

We thank Rosa Maria Arriero for the technical help. This research was supported by Grant FISCAM PI-2006/27 (Gobierno de Castilla La Mancha).

1 DeForge D, Blackmer J, Garritty C, Yazdi F, Cronin V, Barrowman N et al. Fertility following spinal cord injury: a systematic review. Spinal Cord 2005; 43: 693-703.

2 Ibrahim E, Lynne CM, Brackett NL. Male fertility following spinal cord injury: an update. Andrology 2016; 4: 13-26.

3 Leduc BE. Treatment of infertility in 31 men with spinal cord injury. Can J Urol 2012; 19: 6432-6436.

4 Nehra A, Werner MA, Bastuba M, Title C, Oates RD. Vibratory stimulation and rectal probe electroejaculation as therapy for patients with spinal cord injury: semen parameters and pregnancy rates. J Urol 1996; 155: 554-559.

5 Ohl DA, Sønksen J, Menge AC, McCabe M, Keller LM. Electroejaculation versus vibratory stimulation in spinal cord injured men: sperm quality and patient preference. J Urol 1997; 157: 2147-2149.
6 Chung PH, Yeko TR, Mayer JC, Sanford EJ, Maroulis GB. Assisted fertility using electroejaculation in men with spinal cord injury-a review of literature. Fertil Steril 1995; 64: 1-9.

7 Brackett NL, Davi RC, Padron O, Lynne C. Seminal plasma of spinal cord injured men inhibits sperm motility of normal men. J Urol 1996; 155: 1632-1635.

8 Levron J, Aviram-Goldring A, Madgar I, Raviv G, Barkai G, Dor J. Studies on sperm chromosomes in patients with severe male factor infertility undergoing assisted reproductive technology treatment. Mol Cell Endocrinol 2001; 183(Suppl 1): S23-S28.

9 Ohl DA, Denil J, Fitzgerald-Shelton K, McCabe M, McGuire EJ, Menge AC et al. Fertility of spinal cord injured males: effect of genitourinary infection and bladder management on results of electroejaculation. J Am Paraplegia Soc 1992; 15: 53-59.

10 Siosteen A, Steen Y, Forssman L, Sullivan L. Auto-immunity to spermatozoa and quality of semen in men with spinal cord injury. Int J Fertil 1993; 38: 117-122.

11 Becherini L, Guarducci E, Deg|'Innocenti S, Rotondi M, Forti G, Krausz C. DAZL polymorphisms and susceptibility to spermatogenic failure: an example of remarkable ethnic differences. Int J Androl 2004; 27: 375-381.

12 Heruti RJ, Dankner R, Berezin M, Zeilig G, Ohry A. Gynecomastia following spinal cord disorder. Arch Phys Med Rehabil 1997; 78: 534-537.

13 Berezin M, Ohry A, Shemesh Y, Zeilig G, Brooks ME. Hyperprolactinemia, galactorrhea and amenorrhea in women with a spinal cord injury. Gynecol Endocrinol 1989; 3: 159-163.

14 Ohl DA, Sonksen J, Wedemeyer G, Zaborniak MC, Dam TN, Menge AC et al. Canine model of infertility after spinal cord injury: time course of acute changes in semen quality and spermatogenesis. J Urol 2001; 166: 1181-1184.

15 Huang HF, Linsenmeyer TA, Li MT, Giglio W, Anesetti R, von Hagen J et al. Acute effects of spinal cord injury on the pituitary-testicular hormone axis and Sertoli cell functions: a time course study. J Androl 1995; 16: 148-157.

16 Amann RP. The cycle of the seminiferous epithelium in humans: a need to revisit? J Androl 2008; 29: 469-487.

17 Gottschalk-Sabag S, Glick T, Weiss DB. Fine needle aspiration of the testis and correlation with testicular open biopsy. Acta Cytol 1993; 37: 67-72.

18 Batra VV, Khadgawat R, Agarwal A, Krishnani N, Mishra SK, Mithal A et al. Correlation of cell counts and indices in testicular FNAC with histology in male infertility. Acta Cytol 1999; 43: 617-623.

19 Meng MV, Cha I, Ljung BM, Turek PJ. Testicular fine-needle aspiration in infertile men: correlation of cytologic pattern with biopsy histology. Am J Surg Pathol 2001; 25: 71-79. 
20 Mehrotra R, Chaurasia D. Fine needle aspiration cytology of the testis as the first-line diagnostic modality in azoospermia: a comparative study of cytology and histology. Cytopathology 2008; 19: 363-368.

21 Brackett NL, Lynne CM, Ibrahim E, OhI DA, Sønksen J. Treatment of infertility in men with spinal cord injury. Nat Rev Urol 2010; 7: 162-172.

22 Ibrahim E, Brackett NL, Lynne CM. Advances in the management of infertility in men with spinal cord injury. Asian J Androl 2016; 18: 382-390.

23 Kirshblum S, Waring W 3rd. Updates for the International Standards for Neurological Classification of Spinal Cord Injury. Phys Med Rehabil Clin N Am 2014; 25: 505-517.

24 Curt A, Dietz V. Electrophysiological recordings in patients with spinal cord injury: significance for predicting outcome. Spinal Cord 1999; 37: 157-165.

25 Buch JP, Zorn BH. Evaluation and treatment of infertility in spinal cord injured men through rectal probe electroejaculation. J Urol 1993; 149: 1350-1354.

26 Ohl DA, Bennett CJ, McCabe M, Menge AC, McGuire EJ. Predictors of success in electroejaculation of spinal cord injured men. J Urol 1993; 149: 1350-1354.
27 Chung PH, Verkauf BS, Eichberg RD, Casady L, Sanford EJ, Maroulis GB. Electroejaculation and assisted reproductive techniques for anejaculatory infertility. Obstet Gynecol 1996; 87: 22-26.

28 Heruti RJ, Katz H, Menashe Y, Weissenberg R, Raviv G, Madjar I et al. Treatment of male infertility due to spinal cord injury using rectal probe electroejaculation: the Israeli experience. Spinal Cord 2001; 39: 168-175.

29 WHO. WHO Laboratory Manual for the Examination and Processing of Human Semen, 5th edn. World Health Organization: Geneva, Switzerland, 2010.

30 Safarinejad MR. Level of injury and hormone profiles in spinal cord-injured men. Urology 2001; 58: 671-676.

31 Young RJ, Strachan RK, Seth J, Nicol K, Frier BM, Corrall RJ. Is testicular endocrine function abnormal in young men with spinal cord injuries. Clin Endocrinol 1982; 17: 303-306.

32 Hair WM, Gubbay O, Jabbour HN, Lincoln GA. Prolactin receptor expression in human testis and accessory tissues: localization and function. Mol Hum Reprod 2002; 8: 606-611. 